Supporting Information for

\title{
High-Throughput Single-Cell Immunoassay in the Cellular Native Environment Using Online Desalting Dual-Spray Mass Spectrometry
}

Shuting Xu, Jinjuan Xue, Yu Bai*, Huwei Liu

Beijing National Laboratory for Molecular Sciences, Key Laboratory of Bioorganic Chemistry and Molecular Engineering of Ministry of Education, Institute of Analytical Chemistry, College of Chemistry and Molecular Engineering, Peking University, Beijing, 100871, P. R. China

* Phone: +86-10-62758198 (Y. B.).

*E-mail: yu.bai@pku.edu.cn (Y. B.).

This supporting information included the dual nESI setups and desalting results, the characterization of GNP probes, the fluorescence imaging and FCM results of labeled cells, cells monitored in the capillary, and the tandem MS spectra of potential PCs. 
a) Dual nESI 1 (top view)
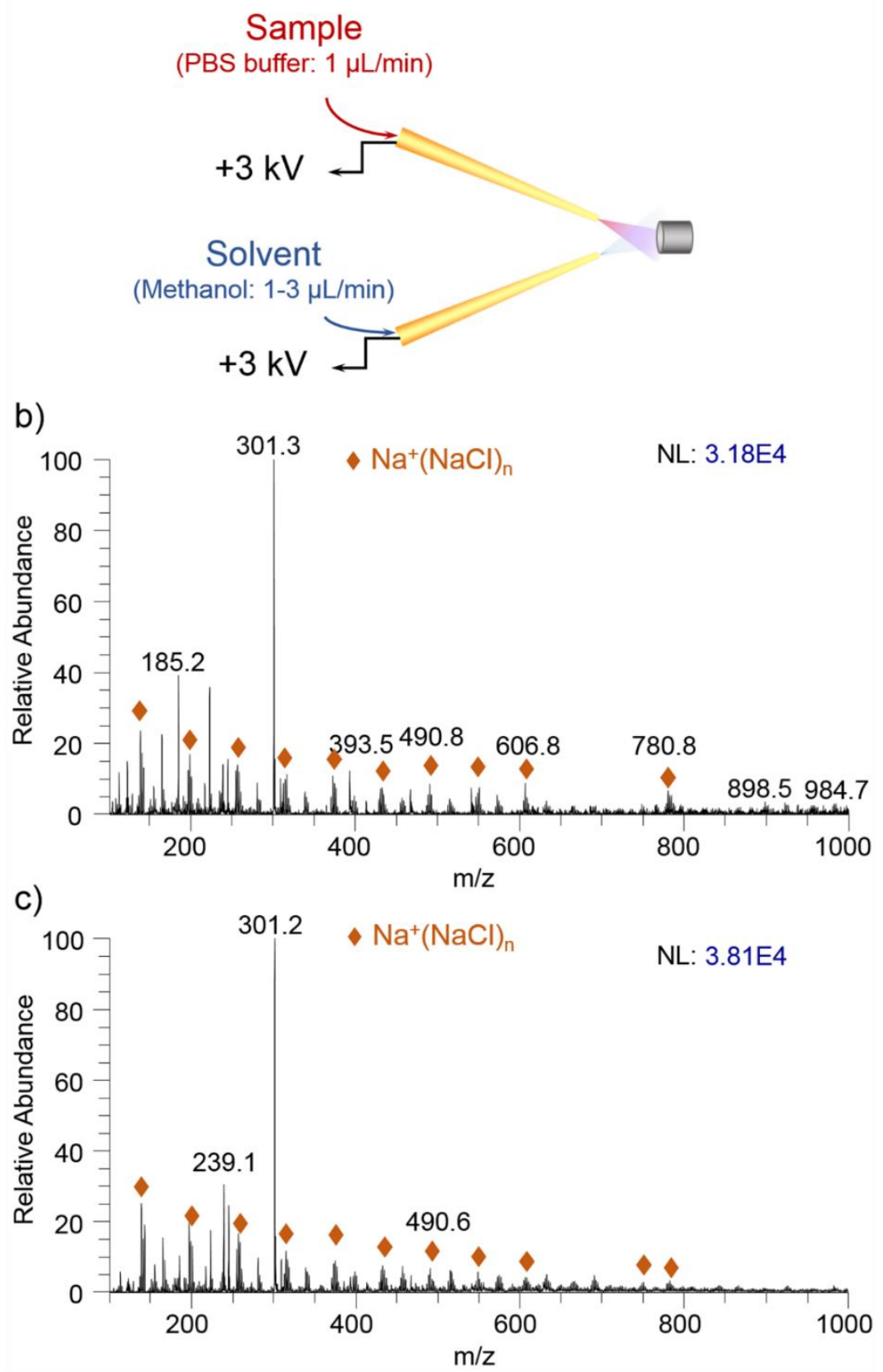

Figure S1. Dual nESI 1. (a) Schematic of the top view of the dual nESI setup with two nESI emitters placed bilateral symmetrically, one nESI for sample with the flow rates of $1 \mu \mathrm{L} / \mathrm{min}$ and another for solvent with the flow rates of $1-3 \mu \mathrm{L} / \mathrm{min}$. (b) Mass spectrum of the GNP probes modified with RMT443 in PBS buffer detected by dual nESI $1(1 \mu \mathrm{L} / \mathrm{min}$ for solvent). Only peaks of salt clusters were detected. (c) Mass spectrum of the GNP probes modified with RMT443 in PBS buffer detected by dual nESI 1 ( $3 \mu \mathrm{L} / \mathrm{min}$ for solvent). Only peaks of salt clusters were detected. 
a) Dual $\mathrm{nESI} 2$ (side view)

\section{Solvent}

(Methanol: 1-3 $\mu \mathrm{L} / \mathrm{min}$ )

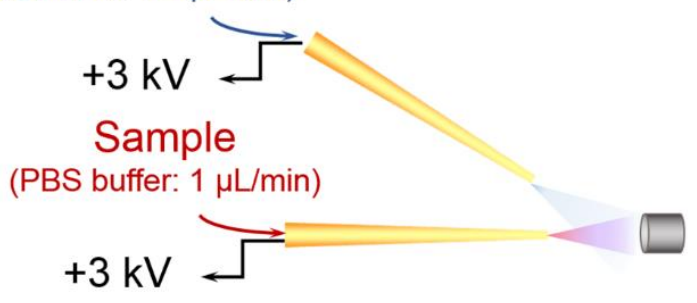

c)

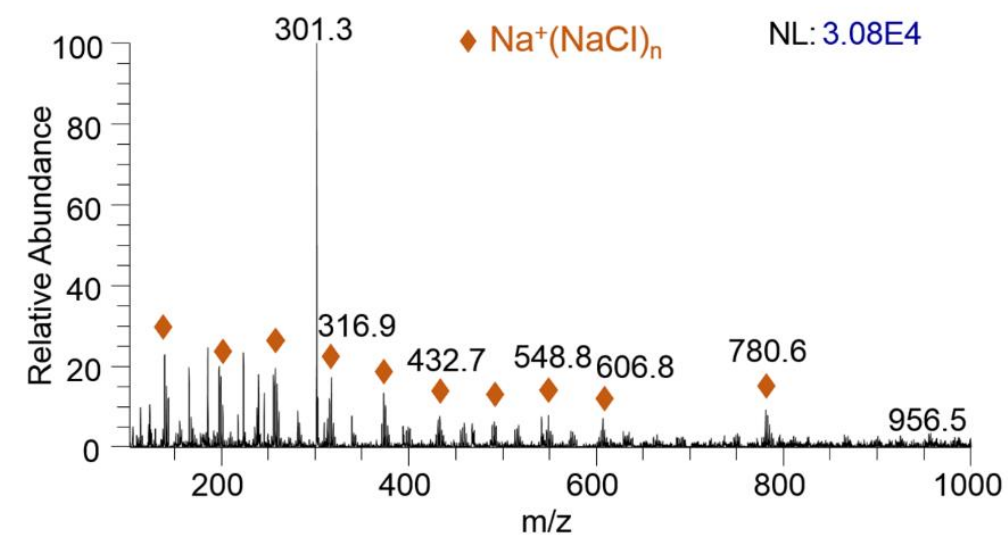

d)

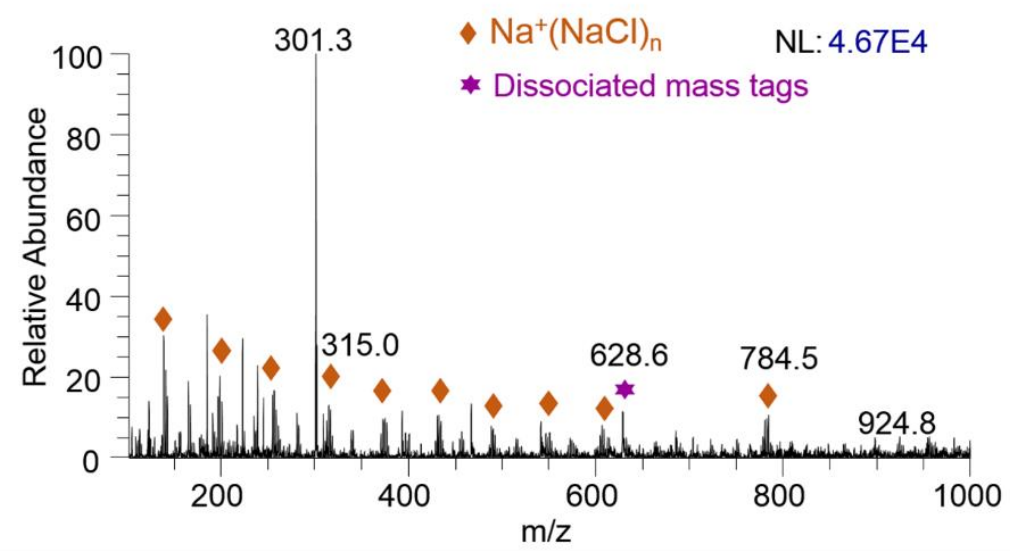

b)

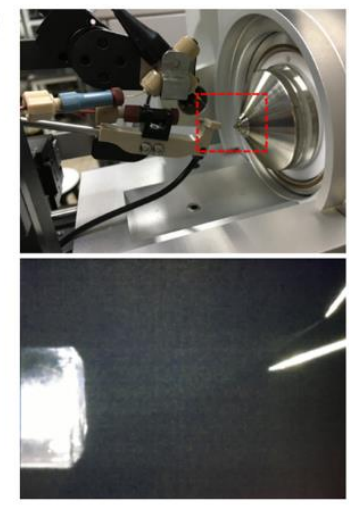

$\mathrm{NL}: 3.08 \mathrm{E} 4$

Figure S2. Dual nESI 2. (a) Schematic and photos of the side view of the dual nESI setup with the up-and-down asymmetric placement of two nESI emitters, one nESI for sample with a flow rate of $1 \mu \mathrm{L} / \mathrm{min}$ and another for solvent with the flow rates of 1-3 $\mu \mathrm{L} / \mathrm{min}$. (b) Photos of the dual nESI 2 setup, and the photo of the two spray $(1 \mu \mathrm{L} / \mathrm{min}$ for sample and $3 \mu \mathrm{L} / \mathrm{min}$ for solvent). (c) Mass spectrum of the GNP probes modified with RMT443 in PBS buffer detected by dual nESI $2(1 \mu \mathrm{L} / \mathrm{min}$ for solvent). Only peaks of salt clusters were detected. (d) Mass spectrum of the GNP probes modified with RMT443 in PBS buffer detected by dual nESI 2 ( $3 \mu \mathrm{L} / \mathrm{min}$ for solvent). Both peaks of salt clusters and dissociated RMT443 were detected. 


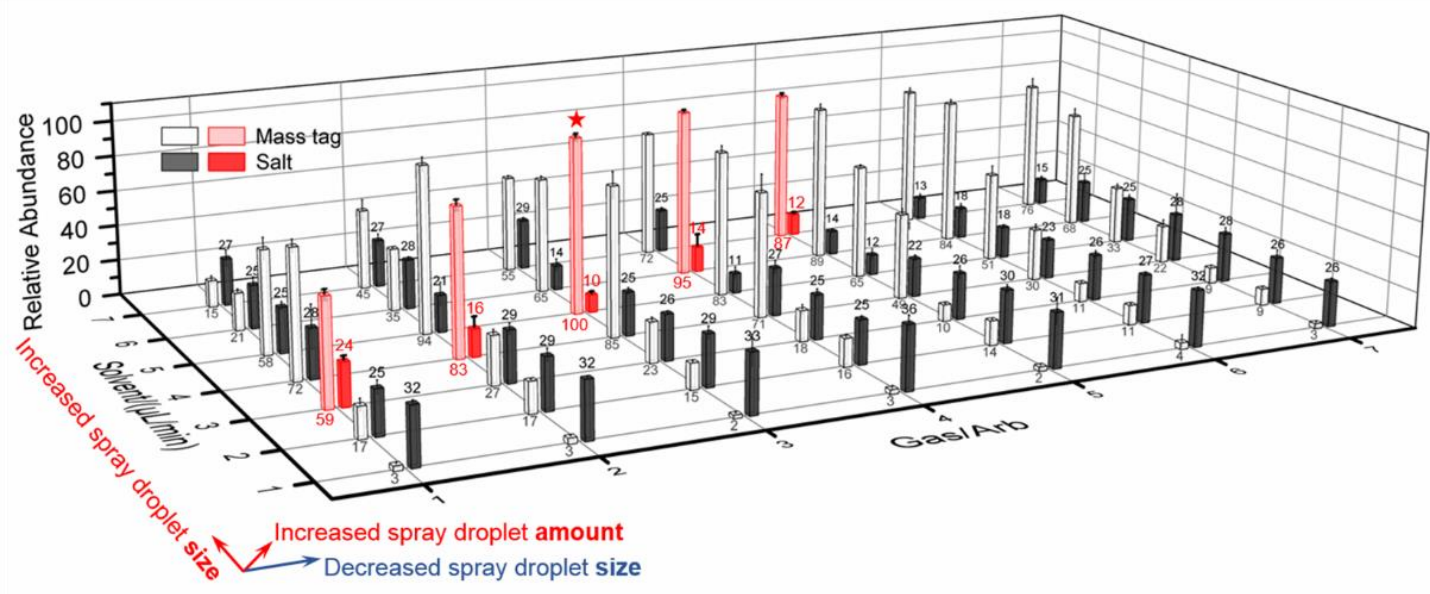

Figure S3. Optimization of solvent flow rates and sheath gas flow rates of ESI in the ESI-nESI setup for online desalting and mass tag detection. Obvious desalting and detection of target mass tags happened when solvent flow rate was larger than $3 \mu \mathrm{L} / \mathrm{min}$ and gas flow rate was larger than 2 arb. Solvent and gas flow rates presented coordinated effects, and representative desalting effects of several solvent/gas flow rates $(3 / 1,4 / 2,5 / 3,6 / 4,7 / 5$; Red bars) were presented in the Manuscript as Figure $3 b$, and $5 \mu \mathrm{L} / \mathrm{min}$ solvent flow rate and 3 arb gas flow rate were finally used for further detection (Marked with red star). The intensities of dissociated RMT443 ions $(\mathrm{m} / \mathrm{z}$ 628.6) and salt clusters ( $\mathrm{m} / \mathrm{z}$ 490.8) were used for evaluation. 
a) ESI off mode

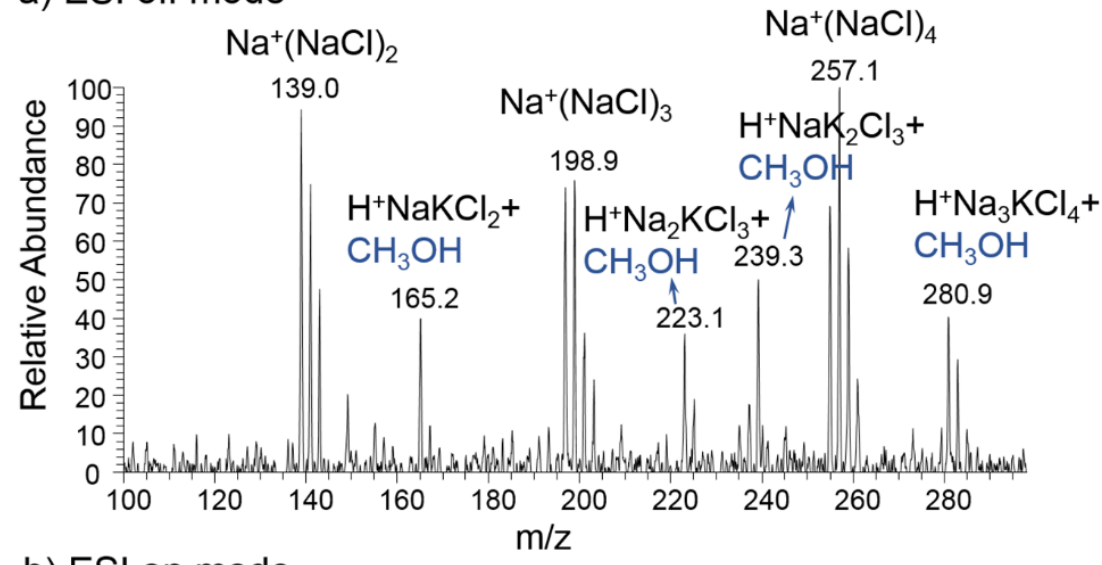

b) ESI on mode

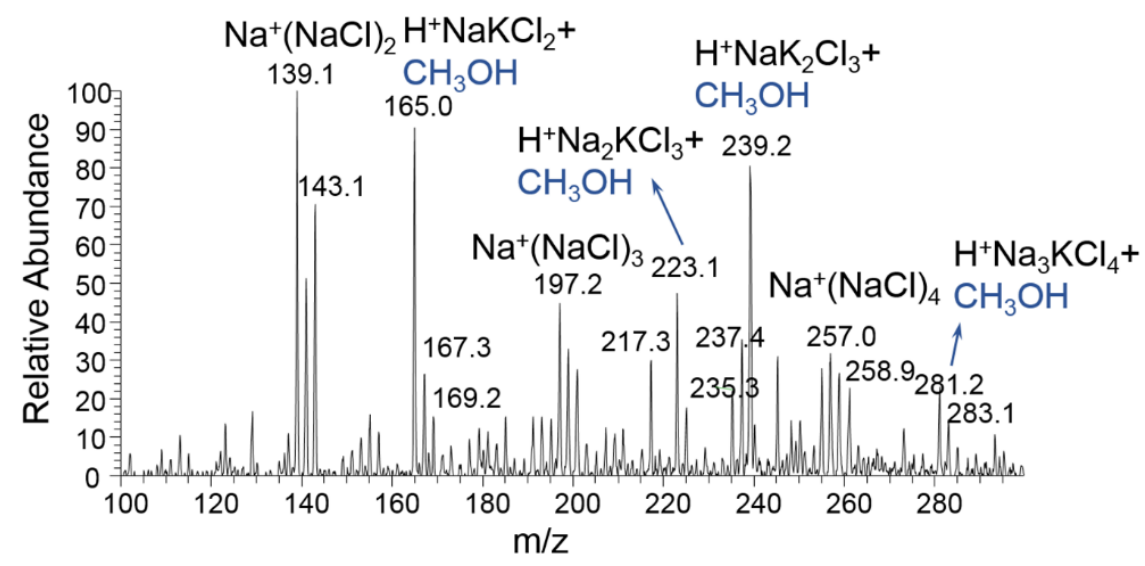

Figure S4. Mass spectra of the GNP probes modified with RMT443 in PBS buffer detected by the (a) ESI off mode and (b) ESI on mode, peaks annotated in blue most likely to be alkali ion-organic solvent adducts, with few evident changes for the ESI on or off modes. 


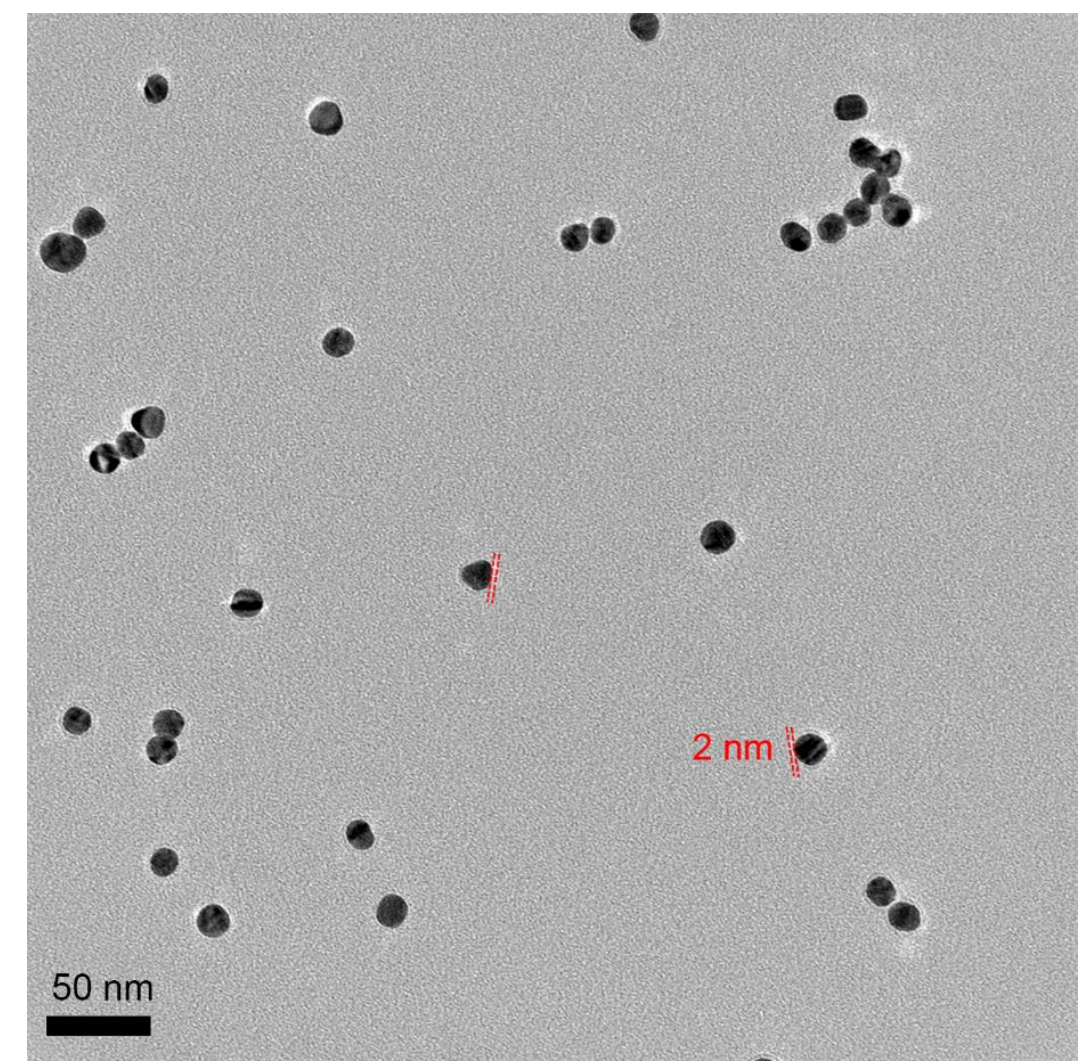

Figure S5. Transmission electron microscopy (TEM) image of antibodies and RMT443 modified GNP probes, suggesting the spherical and monodispersed particles with about $20 \mathrm{~nm}$ in diameter and a modified corona of $2 \mathrm{~nm}$ thickness.
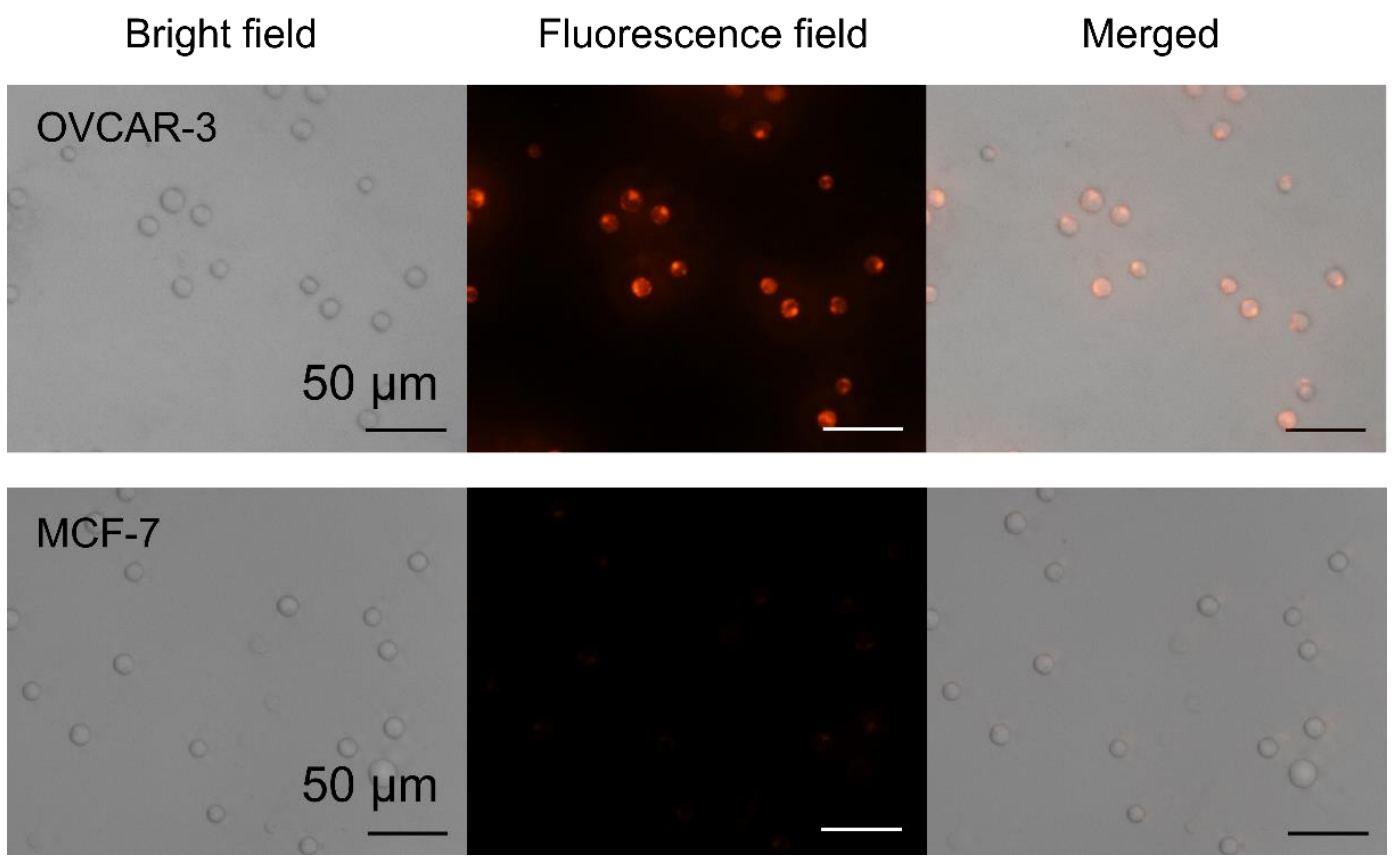

Figure S6. Bright and fluorescence images of the CA125 on OVCAR-3 cells and MCF-7 cells labeled by anti-CA125 and RMT443 modified GNP probes, only the OVCAR-3 cell surfaces were lighted, indicating the high expression of CA125 on OVCAR-3 cells and almost no expression of CA125 on MCF-7 cells. 


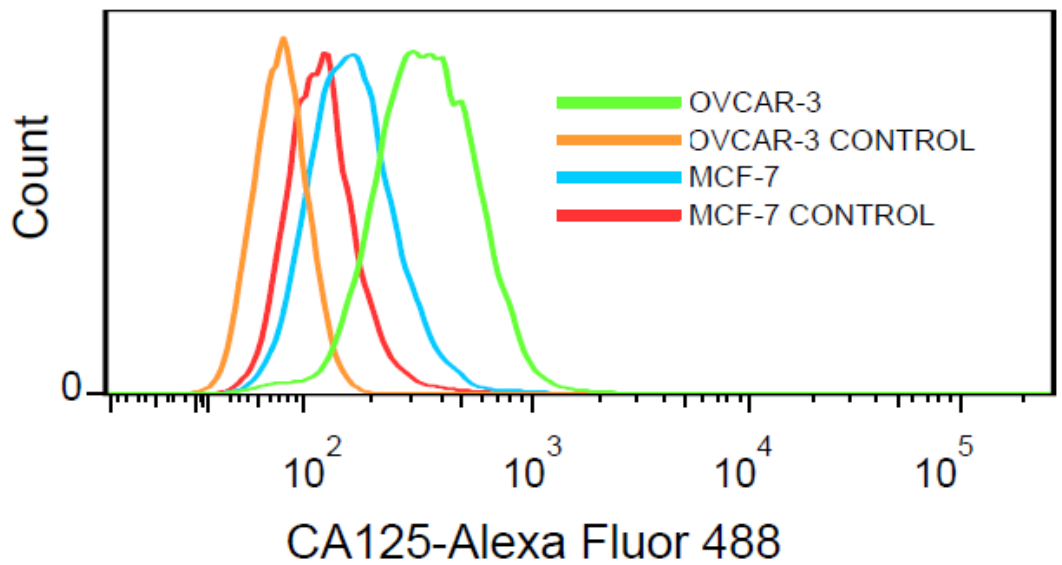

Figure S7. Fluorescence-based flow cytometric results of CA125 on OVCAR-3 cell line and MCF-7 cell line, cells without labeling as control. All experiments were carried out by the detection of at least 10000 cells with the channel FITC/Alexa Fluor $488(530 \pm 30 \mathrm{~nm})$, showing high expression of CA125 on OVCAR-3 cells and almost no expression of CA125 on MCF-7 cells.

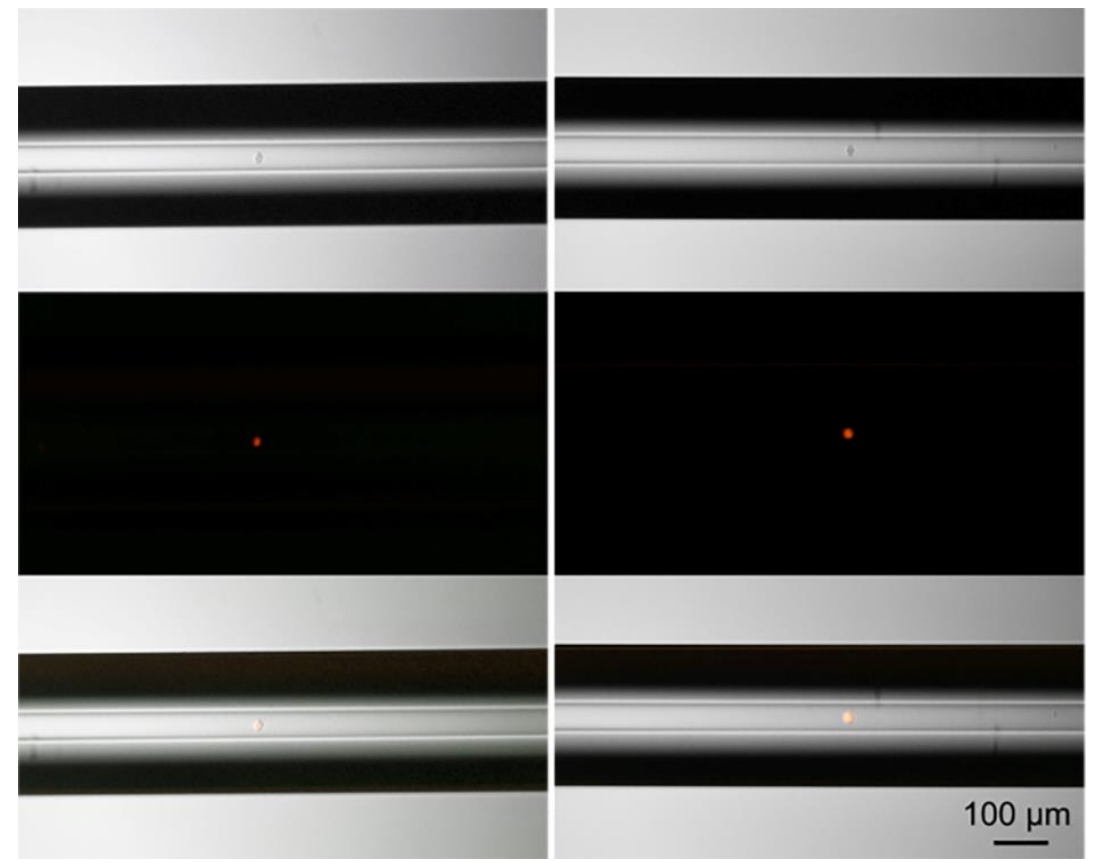

Figure S8. Bright and fluorescence images of OVCAR-3 cells labeled by anti-CA125 and RMT443 modified GNP probes flowing in capillary. Cells in capillary were mostly individual cells with large space between cells. 

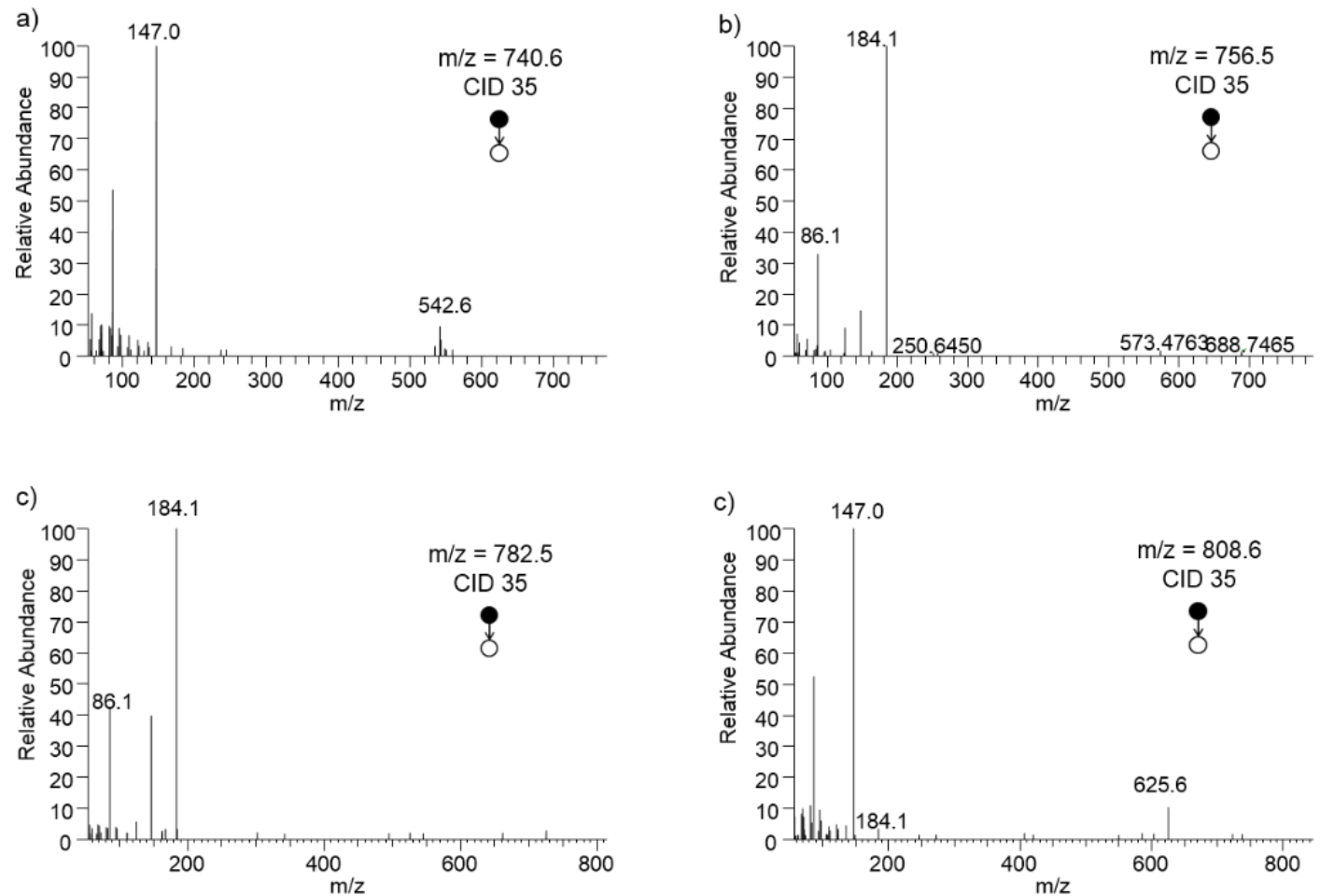

Figure S9. Tandem MS spectra of proposed glycerophosphocholines: $\mathrm{m} / \mathrm{z}$ 740.6, $\mathrm{m} / \mathrm{z}$ 756.5, $\mathrm{m} / \mathrm{z}$ 782.5, and $\mathrm{m} / \mathrm{z}$ 808.6. Collision induced dissociation (CID) with energy of 35 was utilized for the fragmentation. Characteristic fragments of glycerophosphocholines, including $\mathrm{m} / \mathrm{z} 86$ (vinyltrimethylamine), $\mathrm{m} / \mathrm{z} 147$ (sodiated five-member cyclophosphane), $\mathrm{m} / z 184$ (head group of phosphocholines) were detected and used to perform the glycerophosphocholines identification. 